\title{
LA PARTICIPACIÓN EN LA DEFINICIÓN DEL Plan General de Yountville de Halprin. Y UN APUNTE PARTICULAR DE TEATRO
}

The participatory process in the definition of Lawrence Halprin's Urban Plan for Yountville. And a Note on Theater

\author{
Jaume Blancafort \\ Universidad Politécnica de Cartagena, Espanya. \\ Patricia Reus \\ Universidad Politécnica de Cartagena, Espanya.
}

RESUMEN: Lawrence Halprin fue uno de los pioneros en utilizar la participación ciudadana en el proceso de definición del planeamiento, reforma, recuperación y/o diseño urbano, ya en los años 60 en eeuu.

En este texto se describe el proceso ocurrido en la resolución del Plan General de Ordenación Urbana de Yountville, de 1973 a 1975. La utilización de los Paseos de la Consciencia, el Taller Participativo y el teatro para obtener el conocimiento necesario de la ciudadanía para conseguir definir primero las líneas maestras y luego los matices del plan que el pueblo consideraba más conveniente para sus intereses.

El planeamiento resultante consecuentemente enriquecido con el conocimiento y las voluntades que la ciudadanía expresa, y evidentemente diseñado con el rigor técnico necesario, se define mucho más cercano a esta ciudadanía y a la consecución del bien común. En definitiva, se afianza priorizar el interés general sobre el particular.

Palabras Clave: Halprin, Yountville, participación, planeamiento, ciudadanía, proceso, ciudad, teatro. 
RESUM: Lawrence Halprin va ser un dels pioners a utilitzar la participació ciutadana en el procés de definició del planejament, reforma, recuperació i/o disseny urbà, ja en els anys 60 als EUA.

En aquest text es descriu el procés ocorregut en la resolució del pla general urbà de Yountville, de 1973 a 1975. La utilització dels Passejos de la Consciència, el Taller Participatiu i el teatre per a obtenir-hi el coneixement necessari de la ciutadania per a aconseguir definir primer les línies mestres $\mathrm{i}$ després els matisos del pla que el poble considerava més convenient per als seus interessos.

El planejament, resultant conseqüentment enriquit amb el coneixement i les voluntats que la ciutadania expressa, i evidentment dissenyat amb el rigor tècnic necessari, es defineix molt més proper a aquesta ciutadania i a la consecució del bé comú. En definitiva s'aferma prioritzar l'interès general sobre el particular.

Paraules clau: Halprin, Yountville, participació, planejament, ciutadania, urbà, procés, ciutat.

ABSTRACT: Lawrence Halprin was one of the pioneers in using citizen participation in the process of urban definition, reform, recovery and/or design back in the 1960s in the United States.

This text describes the process of implementing the General Urban Plan of Yountville, from 1973 to 1975. Awareness Walks, Take Part workshops and theater were used to gather the necessary knowledge from citizens to first define the guidelines and then the nuances of the Plan that the people considered most suitable for their interests.

The resulting plan, enriched with the knowledge and wishes expressed by citizens, and designed with the necessary technical rigor, comes much closer to these citizens and to achieving the common good. In sum, it prioritizes public over private interests.

KeYwords: Halprin, Yountville, participatory process, urban planning, citizens, city. 


\section{Introducción}

$\mathrm{L}$ awrence Halprin fue un arquitecto americano que desarrolló su práctrabajos se centran principalmente en la arquitectura del paisaje y el planeamiento urbano, especialidad con la que obtuvo un gran reconocimiento profesional, institucional y popular. Sus intervenciones urbanas además de recibir los premios más prestigiosos de las instituciones americanas resultaron focos verdaderos de vida, convirtiéndose en uno de los arquitectos más buscados para diseñar la recuperación urbana de zonas deprimidas y liderar complejos procesos de negociación entre grupos humanos con intereses aparentemente divergentes.

La característica especial que a su forma de planificar le dio especial reconocimiento, y motivo de la investigación en la que surge este artículo, fue la utilización de los procesos participativos, y los talleres Take Part (Participa) en los que involucraba a la población.

Los procesos participativos los empezó a utilizar en los años 60 en colaboración con su mujer, Anna Halprin, una de las pioneras de la danza contemporánea, y los fue perfeccionando y adaptando para entender y aplicar al planeamiento las voluntades de la ciudadanía implicada.

La concepción se basa en una convicción profunda:

Creemos en la trascendente influencia que tiene el entorno en las vidas de las personas y la importancia de la necesidad de involucrar a éstas para que este entorno no sea diseñado unilateralmente por otras. (Halprin, 1972 a)

Para ello, mientras desde su oficina se empezaba a trabajar en el último plan encargado, ya se preparaban los talleres ciudadanos donde implicarían a las comunidades afectadas. El objetivo era dar voz a todos aquellos que no formando parte de ningún grupo de poder eran precisamente los más afectados por las decisiones que se tomarían en despachos ajenos a la comunidad.

Así, entendían los talleres participativos como:

[...] talleres que permiten a la gente tomar decisiones sobre cómo y dónde van a vivir. (Halprin, 1972 b) 
Pero en los talleres también hacía participar a los políticos, burócratas, representantes electos, representantes del poder económico, etc. e implicándolos, haciendo grupos donde se mezclaban procedencias, niveles económicos y culturales, y conduciendo nuevas empatías entre ajenos, conseguía obtener consensos en ideas, voluntades, anhelos de los ciudadanos para y por su ciudad; que posteriormente traducía técnicamente para hacerlos viables.

Con ello no sólo obtenía las bases para realizar unos proyectos adecuados a la ciudadanía, escuchando un amplio espectro de intereses, muchos de los cuales sin el proceso participativo nunca hubieran podido ser atendidos, sino que además gracias a la implicación de los ciudadanos, cuando luego se ejecutaban los proyectos éstos eran recibidos con entusiasmo.

La oficina de Lawrence Halprin trabajó en ciudades grandes y pequeñas, en proyectos de nuevas planificaciones urbanas, en recuperación de centros históricos degradados, en plazas, parques y calles a lo largo y ancho de los EEUU. Destacan proyectos para San Francisco, Portland, Nueva York, Washington o Denver, pero en el aprendizaje del método participativo entre los años 60 e inicios de los 70 estuvo ensayando en lugares más pequeños y menos conocidos pero que influenciarían definitivamente su forma de proceder.

Como se apunta en el artículo «Pioneros de la participación colectiva en los procesos de planificación urbana» donde se introduce el trabajo de Anna y Lawrence Halprin y se describe un marco conceptual y práctico de la participación ciudadana en la construcción de la ciudad:

La pareja Halprin desempeña un papel trascendental en la evolución conceptual tanto del planeamiento urbano como de las artes escénicas y ejemplifica una concurrencia paradigmática de la arquitectura y la danza. Este cruce singular, muy poco documentado en la Europa continental y prácticamente nulo en lengua española, nos aporta el conocimiento de posibles alternativas ya comprobadas con acierto en la construcción participada de la ciudad. (Blancafort, 2015)

El que a continuación se describe fue el proceso seguido para la redacción del nuevo Plan Urbano de Yountville, un pequeño pueblo del Valle de Napa en California. 
Uno de los aspectos más curiosos y destacados del proceso ocurrido allí fue la utilización del teatro como medio de transmisión de las diferentes ideas urbanas que surgieron en el taller participativo.

A nivel metodológico apuntar que esta revisión se fundamenta en los documentos originales producidos en ese momento y conservados en la $\mathrm{Law}$ rence Halprin Collection (LHC), en los Architectural Archives de la University of Pennsylvania; que se han ordenado cronológicamente para construir el relato y poder comprender dicho proceso.

\section{El contrato de servicios arquitectónicos. Política y ciudad}

Según leemos en el informe que la consultoría económica $\mathrm{GG}+\mathrm{A}$ redacta para Lawrence Halprin en noviembre del año 1972 (LHC, 1972), la población de Yountville, una comunidad de unos 5000 habitantes en el valle de Napa, California, se encontraba en un punto de inflexión.

Yountville era una pequeña y encantadora ciudad semi-rural, cuyo grupo poblacional mayor era el de los residentes del Hospital de Veteranos de California. Sus comercios habían sabido aprovechar la gran cantidad de turistas que atraía la industria del vino, y por otro lado, debido a su dimensión y emplazamiento había recibido un considerable número de familias cansadas de la vida urbana que habían encontrado allí un buen lugar donde vivir. Pero en 1972, había una alta presión para desarrollar nuevos proyectos comerciales y promociones residenciales, con lo que el gobierno de la ciudad consideró necesario dirigir el crecimiento futuro de la ciudad, antes de que la comunidad sufriera cambios indeseables y quizás irreversibles.

Como nos confirma un informe sobre la población del Departamento de Conservación, Desarrollo y Planeamiento del condado de Napa, Yountville tenía un crecimiento poblacional anual de $+8,70 \%, 3$ puntos por encima del ranking de la siguiente población con más crecimiento del valle (LHC, 1973 a).

Así, conociendo la intención del Ayuntamiento de Yountville de querer controlar la situación antes de que el mercado decidiera por ellos y afectara a la vida de todos sus habitantes, y sabiendo del rastreo de posibles candidatos para conducir esa tarea que les resultaba de vital importancia, la oficina de LH\&A ofrece sus servicios. 
Lawrence Halprin \& Associates está interesado en ser considerado por el Ayuntamiento de Yountville para ofrecer servicios profesionales.

Aunque su comunidad es pequeña, los problemas que afrontan son los mismos a los que se enfrentan las comunidades en todo el estado. Estamos bien calificados para trabajar con su comunidad en pos del desarrollo de una solución coherente a los problemas de crecimiento y cambio. Creemos que en el marco de la comprensión del entorno natural podemos ayudar a la comunidad en la búsqueda de formas para mantener y mejorar el estilo de vida que sus residentes tienen y están buscando. (LHC, 1973 b)

Con el ofrecimiento de sus servicios adjuntan una planificación de cómo ellos plantearían organizar el estudio del nuevo Plan de Yountville. En este propuesta, además de los prescriptivos puntos para el desarrollo del nuevo plan urbanístico, cabe destacar unas aportaciones que junto a su currículum, resultarán decisivas para que el Ayuntamiento se decante por Halprin. Proponen tres reuniones con los vecinos en momentos claves de la toma de decisiones del plan; en las fases que corresponden con: III - Formulación de Metas y Objetivos; VII - Desarrollo de la Estrategia de Implementación y VIII - Preparación del Informe Final.

Haciendo así partícipes a todos los habitantes de Yountville de la toma de decisiones que afectarían sus vidas en la futura evolución de la población.

Finalmente en junio de 1973, el Ayuntamiento de Yountville, después de haber valorado la posibilidad de contratar a diversos candidatos, decide decantarse por la firma de LH\&A y le propone usar el planteamiento que ofrecía por carta como base para redactar el contrato.

En sus valoraciones destacan dos puntos que califican no solamente la elección del profesional sino al propio Ayuntamiento:

- La firma seleccionada tiene que ser consciente de las cualidades únicas de la comunidad y estar atenta a estas cualidades con el fin de que el estilo de vida rural de Yountville pueda continuar incluso cuando se experimente el cambio de desarrollo. 
- El coste no es un «gasto», sino una «inversión»; una inversión que muy satisfactoriamente determinará la calidad de vida en Yountville para el futuro previsible. (LHC, 1973 c)

El primer punto reitera el sentimiento de la calidad de vida reinante y la voluntad de mantenerla, con lo que denota la consciencia del Ayuntamiento en relación a lo que están decidiendo. El segundo confirma el hecho de decidirse no por la oferta más barata sino por la mejor cualificada.

Desde el día en que se manifiesta en acto público la voluntad de contratar a Halprin hasta la fecha de la firma del contrato, el Ayuntamiento le avanza información existente al respecto del planeamiento vigente: planos urbanos, de servicios, mapa geológico, requerimientos del gobierno regional, voluntades, etc. y a la vez van negociando el contrato con base en la oferta esquemática que había pasado meses antes.

En julio de 1973, LH\&A envía al Ayuntamiento un borrador del contrato con todos los capítulos de la programación del proceso de proyecto del Plan. En dicho borrador aparecen todos los puntos que hacen referencia a los pagos, fases del proyecto, documentación a entregar, calendario, etc. Llama la atención el último punto de la «Sección II, Alcance de los Servicios». Con este punto LH\&A se asegura el compromiso del Ayuntamiento a dejar por escrito la aceptación de los resultados de los talleres participativos; eliminando la posibilidad de convertir los talleres ciudadanos en una mera operación de maquillaje o distracción.

\footnotetext{
La aparición de LH\&A EN REUNIONES PÚBLICAS SE LIMITARÁ A TRES, UNO EN LA FINALIZACIÓN DE LA FASE III y respectivamente en la finalización de las Fases VII y VIII para revisar fases completadas hasta esa fecha. Las aprobaciones para proceder a las fases IV y VIII serán remitidas por el Ayuntamiento dentro de dos semanas de las sesiones públicas mencionadas anteriormente. (LHC, 1973 d)
}

Y por si alguien aún pudiera tener alguna duda del compromiso de los talleres en la planificación urbana se añade:

SECCIÓN III. TIEMPO DE EJECUCIÓN:

Todo el proyecto del Plan General de Yountville - 1973 se completará dentro de los seis meses a partir de la fecha de ejecución de este contrato. Si las aprobaciones del Ayuntamiento, que se pidieron en la Sección II, no pudieran realizarse en las dos semanas siguientes a las sesiones públicas designadas, se ampliará automáticamente el tiempo en la programación de entregas. (LHC, 1973 e) 
Finalmente, en agosto de 1973 se firma el contrato para redactar el nuevo Plan General.

\section{Inicio del proceso participativo. Consciencia del lugar y deseos}

Una vez firmado el contrato LH\&A manda al Ayuntamiento una programación detallada de lo que se prevé que realizarán durante los siguientes meses y propone una fecha para organizar el primer taller participativo con la población, el que hace referencia a la Fase III, correspondiente a la formulación de metas y objetivos.

Seguidamente y en carta de septiembre de 1973, desde el Ayuntamiento plantean varias dudas al respecto de este futuro taller que indican cierta incertidumbre hacia el «experimento participativo» pero el alto grado de compromiso asumido:

El Ayuntamiento, junto con un número de ciudadanos interesados, se ha reunido la noche del martes 04 de septiembre con el fin de formar un comité de ciudadanos para trabajar con su empresa durante el proceso de planificación.

Un comité parcial, consistente en el consejo y otros trece ciudadanos, ha sido nombrado.

El comité reconoce que necesitará ampliar este grupo para facilitar la participación de una base más amplia de la comunidad y que lo hará en el futuro con su ayuda. Sus miembros convienen en que es necesaria una fecha de reunión permanente y seleccionan el primer y tercer martes de cada mes a las 7:30 de la tarde.

[...] el grupo considera que sería oportuno tener alguna indicación para nuestra próxima reunión el 18 de septiembre para que así podamos prepararnos para el día señalado del taller.

Además, háganos por favor comentarios en cualquier momento con respecto a este comité, su papel, o alguna sugerencia que pueda tener. Le aseguro que esto sería útil y apreciado.

[...] el grupo siente la necesidad de saber cómo resultaría la experiencia del taller, Qué estaríamos haciendo, etc., y qué se podría esperar de llevar a cabo esta experiencia [...] (LHC, $1973 \mathrm{f})$

Mientras, en LH\&A, además de ir contestando y dando instrucciones al comité de ciudadanos encargado de preparar el día del taller, va desarrollando las Fases I y II correspondientes a la recopilación de los antecedentes históricos, normativos, etc. y la identificación de la escala y tipo de oportunidades económicas que tiene y/o puede desarrollar Yountville. 
Por otra parte, Jim Burns de LH\&A hace un viaje de reconocimiento a Yountville para preparar tanto las instrucciones del Paseo de la Consciencia como lo que trabajarán en el taller participativo; comprobar la adecuación de las dimensiones del local que el Ayuntamiento ha propuesto para desarrollar el evento y tomar fotografías de la ciudad y su entorno que luego podrán usarse para proyectar durante la sesión pública.

Para animar a la participación, además de la difusión boca a boca que tan bien funciona en una población pequeña y el reparto de octavillas para dicho fin (fig. 1), el 25 de septiembre el alcalde publica un comunicado en el periódico local en el que, aprovechando las felicitaciones y agradecimientos en relación a la fiesta organizada para la recuperación del camión de bomberos, los emplaza a implicarse en el importante reto que tiene el pueblo, que es la definición del nuevo Plan.

Se transcriben a continuación unas líneas del comunicado del alcalde porque transmiten directa y claramente la filosofía del quehacer de Halprin:

El Plan es una necesidad y es algo que debemos hacer para nuestro propio bien [...]

El Comité de Planificación está trabajando, y espero que cada uno de vosotros participe de alguna forma u otra, porque necesitamos vuestra ayuda y orientación. Además, como todos tenemos una idea de lo que implica un plan, pronto tendréis la oportunidad de participar en lo que se llama un Paseo de la Consciencia de la Comunidad $[\ldots]$

En efecto, nuestros planificadores [...] quieren saber más acerca de Yountville y lo que cada uno de vosotros quiere que sea la comunidad.

En realidad lo que va a pasar es que todo el que quiera podrá participar en un paseo por la ciudad y observar y discutir las cosas que son más importantes.

A veces me pregunto cuántos de nosotros nos tomamos tiempo para apreciar lo mucho que Yountville tiene que ofrecer; creo que todos estamos de acuerdo en que si somos más conscientes de nuestro entorno y de algunos problemas relacionados con la construcción de la ciudad, habrá menos tendencia a destruir aquello en lo que creo que todos estamos de acuerdo, es decir, la agradable atmósfera de nuestro pequeño pueblo-rural. (LHC, $1973 \mathrm{~g}$ ) 

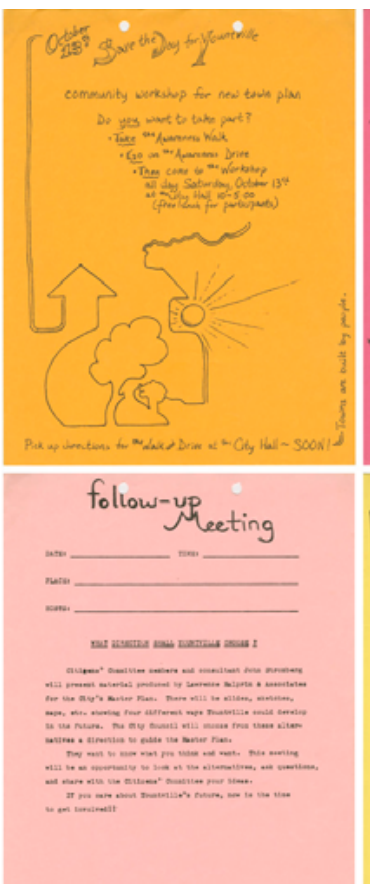
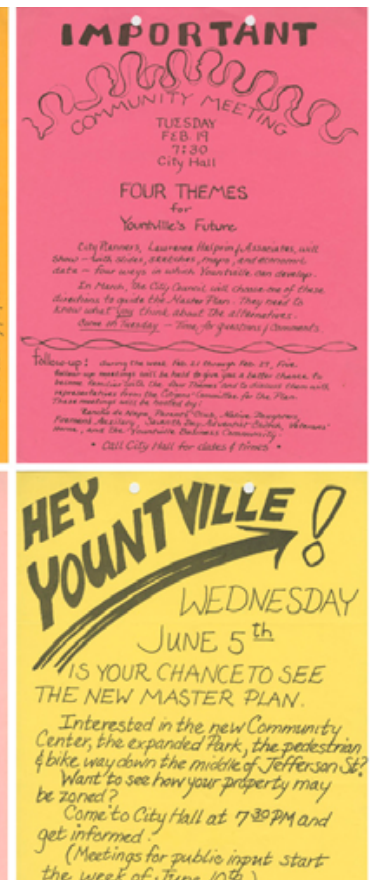
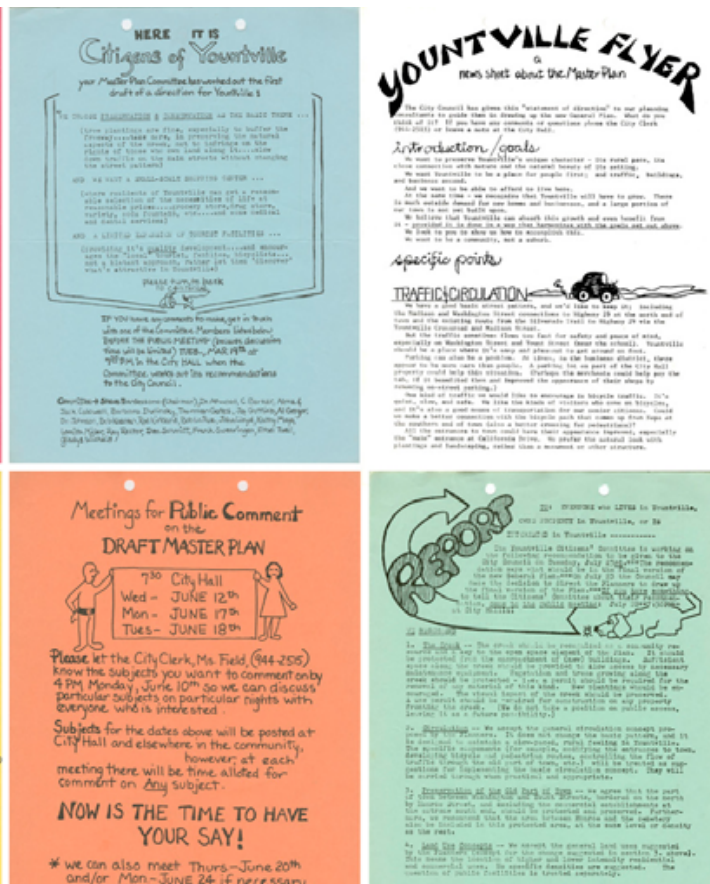

Figura 1. Recopilación de distintas octavillas para promover la participación de los ciudadanos de Yountville en los diversos actos participativos. Fuente:

Lawrence Halprin Collection, The Architectural Archives, University of Pennsylvania [Folder: 014.I.B.1249]

Un par de semanas antes del esperado evento público, desde LH\&A escriben al alcalde dándole explicaciones detalladas de qué y cómo va a desarrollarse dicho taller y adjuntándole cientos de copias con las instrucciones y mapas para que los ciudadanos puedan realizar el Paseo de la Consciencia cualquiera de los días previos al taller, y conseguir así que el día señalado todos los participantes trabajen con un conocimiento base.

También se adjuntan las instrucciones fundamentales para poder participar en el taller.

E1 9 de octubre se publica en el periódico local el comunicado del alcalde:

Reserva el Día de Yountville.

El sábado, 13 de octubre pertenece al pueblo de Yountville y a cualquier otra persona que esté interesada en el futuro de la ciudad.

Tu participación en el Paseo de la Consciencia esta semana y el taller del sábado de 10 a. m. a 5 p. m. ayudará a determinar el futuro de Yountville. Será tu oportunidad de tomar 
un papel activo en el proceso de planificación y compartir tus pensamientos e ideas con tus vecinos.

La jornada se titula «Reserva el Día de Yountville», espero que todo aquel interesado en lo que pasa en la población tendrá tiempo para invertir al menos un día en el futuro de la ciudad. A veces es tentador no hacer nada con la esperanza de que no va a cambiar la situación; pero hoy en día hay una amplia evidencia que indica que el especulador siempre está listo para actuar si bajamos la guardia. Creo que no hace falta decir que nadie planea un edificio sin saber el uso que tendrá; o dicho de otra manera, si no sabes a dónde vas, terminarás en algún otro lugar. En efecto, no comenzamos con la planificación; comenzamos con los objetivos o necesidades; éstos deben ser identificados primero por las personas involucradas y no sólo por el consejo o el comité de planificación.

No hace falta ser un experto para preguntarte si prefieres vivir en una comunidad agrícola como Yountville o una comunidad suburbana. Si Yountville tiende hacia una densidad y estilo de vida que te gusta o no, entonces danos tu opinión el sábado. Vosotros, las personas que residís aquí, debéis seleccionar los objetivos finales; ¡y eso es precisamente lo que nosotros y nuestro arquitecto, Lawrence Halprin \& Asociados queremos que hagáis!

El sábado es tu día en el Ayuntamiento; así que espero que te unas a nosotros. Habrá almuerzo al mediodía. (LHC, 1973 h)

El paseo en cuestión se puede hacer en una mañana y se indica un recorrido e instrucciones a seguir en puntos determinados de la ciudad, (LHC, 1973 i) como:

- $\quad$ En este cruce cierra los ojos durante un minuto y apunta los sonidos que has escuchado.

- Observa el uso de la carretera aquí.

- ¿Qué pasa en los terrenos a cada lado de la iglesia?

- Imagínate un uso para este espacio.

- ¿Dónde juegan los niños en este barrio?

- Mira a ambos lados de esta calle, lo más lejos que puedas, y describe que ves.

- Imagínate este lugar en Diciembre y descríbenos como te gustaría que fuese.

- A las 12:15 estés donde estés, ve al restaurante, café o tienda de comestibles y almuerza sin gastarte más de $2 \$$; conversa con la persona que te atienda y pregúntale sobre cómo le gustaría que fuera Yountville en un futuro.

Evidentemente, todas estas acciones no son gratuitas, están preparadas para hacer reflexionar sobre la ciudad, poner en cuestión lugares, ensalzar sitios, imaginar alternativas, y en definitiva hacer consciente a la ciudadanía del entorno donde residen, para poder trabajar luego sobre ello. 


\section{Taller - Reserva el día de Yountville}

Aunque la mayoría de los participantes habían hecho el Paseo de la Consciencia los días anteriores, hay un pequeño grupo de gente que lo hizo ese mismo día temprano por la mañana, para luego poder participar a partir de las 10 en el taller.

El taller (fig. 2) se desarrolla en un solo día, el sábado 13 de octubre de 1973 y tiene el siguiente programa:

9:30-9:45 Reunión en el Ayuntamiento del equipo que dirigirá el taller.

10:00 Introducción del Alcalde y LH\&A.

Programa I

10:20-11:20 Se hacen 7 grupos de trabajo sacando los números de los participantes de un sombrero y en cada uno se comparte y discute qué encontraron-descubrieron-observaron en el Paseo de la Consciencia.

$1 / 2$ hora para discutir sobre el tema.

$1 / 2$ hora para preparar un dibujo, una declaración, un poema, un collage para contar en un par de minutos una idea para compartir con todos.

11:20-11:30 Presentación de la idea representativa de cada grupo.

Almuerzo

Programa II

1:30-3:00 Los mismos grupos trabajan durante 1 1/2 horas en qué les gustaría que fuera Yountville en un futuro.

$1 / 2$ hora para preparar la principal idea para compartir con todos.

3:00-4:00 Presentaciones de los grupos.

4:00-5:00 Cierre. Lawrence Halprin realiza un informe oral de lo que ha sucedido en esta jornada y la previa del paseo y traza ciertas conclusiones sobre los deseos e ideas aparecidas.

También se explica brevemente qué sucederá con el proceso a partir de ahora, y cuáles van a ser los siguientes pasos. (LHC, 1973 j) (LHC, 1973 k) 


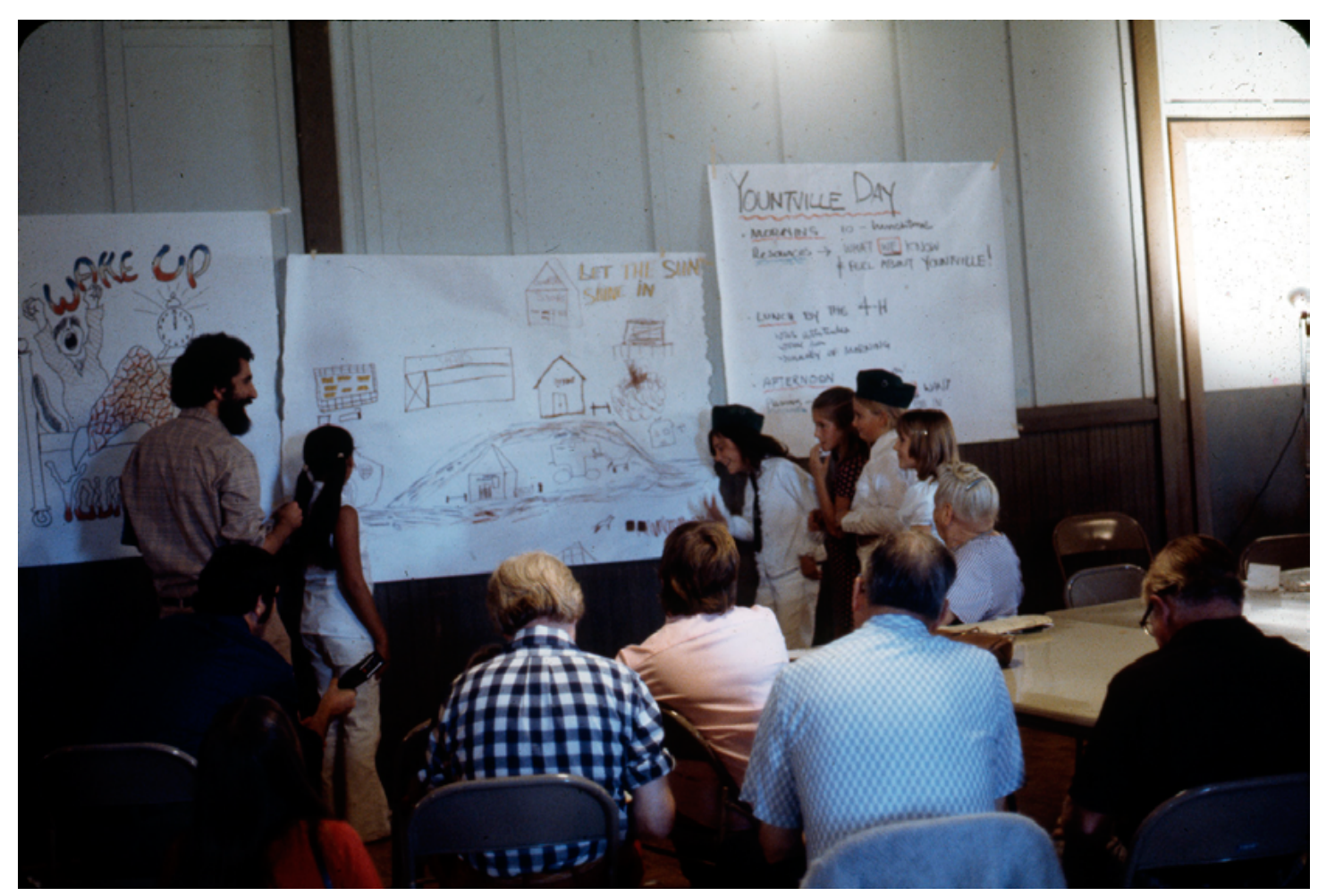

Figura 2. Imagen del Taller Take Part en Yountville, 1973. Fuente: Lawrence Halprin Collection, The Architectural Archives, University of Pennsylvania [Slide: 014.VI.HC-324]

A continuación se transcriben fragmentos del registro telegráfico que el equipo de LH\&A iba anotando constantemente en el cuaderno del taller:

- Jim Burns (LH\&A) hace la descripción de lo que los participantes van a realizar en las siguientes horas y termina diciendo: «que vosotros compartiréis con todos nosotros durante dos minutos al final de la siguiente hora». [...] Inmediatamente los grupos entran en acción.

- Bolígrafos, fieltros, marcadores, rotuladores, gomas, tabletas de escritura, ceniceros, etc. estaban listos para este fin.

- Los técnicos de la firma LH\&A se distribuyen entre las distintas mesas para ayudar a los grupos a interpretar los resultados, obtener materiales y guardar notas. Se les permite sentarse entre los participantes.

- Como en la mayoría de los talleres, los participantes empiezan a usar el lenguaje corporal y otros dispositivos para trasladar al resto de miembros del grupo sus sentimientos personales sobre Yountville.

- Después de media hora los grupos de discusión comienzan a trabajar para preparar una exposición verbal y gráfica de sus descubrimientos (fig. 3). 
- Muchas personas que no están acostumbradas a usar las artes gráficas colocan todos los materiales que pueden junto a una hoja grande de papel de estraza y esperan en silencio a que algo suceda.

- En un primer momento un individuo valiente dibuja una línea serpenteante por el centro de la hoja.

- Alguien prueba un rotulador para ver de qué color es, y ésta es la señal para que alguien más se lance.

- Pronto aparecen muchos intereses confrontados. Entonces todo el mundo quiere intervenir.

- Plumas y manos vuelan en todas direcciones.

- Ciertos miembros del grupo intentan hablar de forma individual con un técnico, pero esto provoca la intervención del resto.

- Algunas personas incluso llegan a dibujar una línea en el centro de su mesa para limitar el acceso a otros miembros. Unos se quedan sin habla, pero a menudo los conflictos se resuelven cuando un miembro muestra la puerta a otro.

$[\ldots]($ LHC, 1973 l)

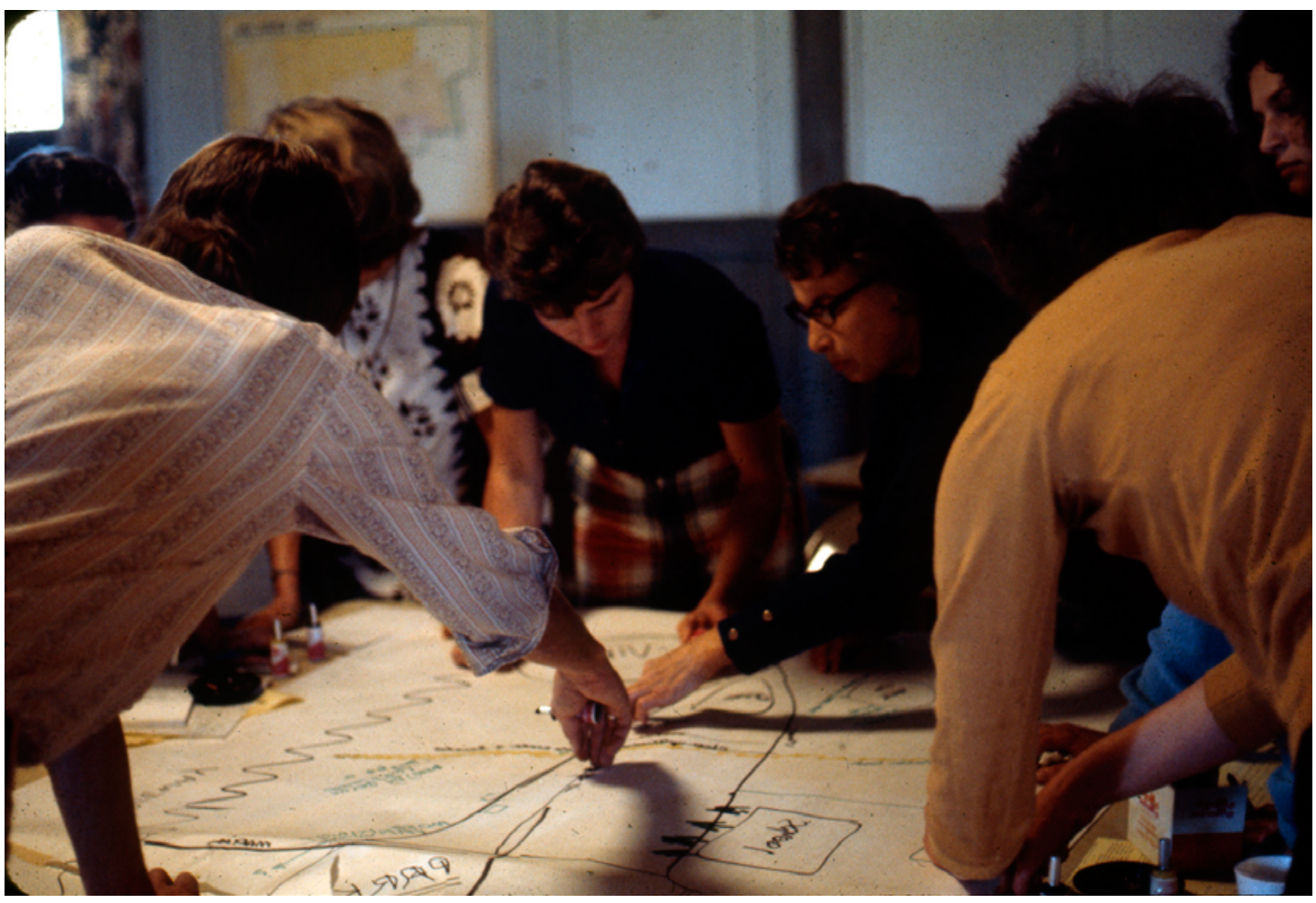

Figura 3. Imagen del Taller Take Part en Yountville, 1973. Fuente: Lawrence Halprin Collection, The Architectural Archives, University of Pennsylvania [Slide: 014.VI.HC-326] 
La mayoría de las presentaciones de los grupos estuvieron relacionadas con los equipamientos que los ciudadanos querían tener en la ciudad: centro cívico y social para adultos y niños, un espacio interior de recreo (piscina, baile...), centro de actividad nocturna y entretenimiento, un mercado, un pequeño museo histórico, un centro de visitantes, una biblioteca, un parque para ancianos, viviendas baratas para granjeros jóvenes, tiendas de cercanía..., pero también hubo algunas propuestas más conceptuales como que Yountville necesitara conservar su carácter del Oeste, la creación de una ruta nupcial o la necesidad de un nuevo despertar de Yountville.

Para cerrar el taller, Lawrence Halprin hizo una revisión de las ideas que habían surgido durante todo el día: unir a la gente, conservación, preservación, árboles y naturaleza, zonificación mixta, atmósfera rural, entretenimiento, soluciones al tráfico, arquitectura heterogénea, edificios bajos, lugar humano, los asuntos de los residentes por encima de los de los turistas para mantener la calidad de vida, etc.

Para terminar el día todos salieron fuera del ayuntamiento para ver el atardecer y hacerse un par de fotos de grupo.

En los días posteriores al evento numerosas noticias aparecieron en los periódicos comentando el taller, entrevistando a gente que había participado y haciéndose eco del revuelo que éste había provocado en la actitud de la gente.

Se anotan unos breves apuntes de tres noticias distintas que aparecieron en el Napa County Records para entender no sólo la dimensión de la acción, sino aportar también algunos datos que no se habían encontrado entre los documentos archivados de LH\&A.

Bienvenido a Yountville

Un renacimiento encantador está ocurriendo en este pueblo de bolsillo.

No sólo los residentes de la ciudad, sino también gran parte del Napa Valley está mirando con avidez el progreso y discutiendo sus alternativas.

La gran pregunta ahora es: ¿Hacia dónde nos dirigimos? (LHC, 1973 m)

El Día de la Conciencia

Con todo, el sábado fue uno de esos días en los que la gente se potencia. Nunca he visto tal espíritu y entusiasmo. Los niños del $4 \mathrm{H}$ bajo el liderazgo de la señora Ulrich hicieron un gran trabajo en el almuerzo, por no mencionar el entusiasmo que Bob Zaro y sus maestros 
pusieron llevando a los alumnos de primero a sexto grado a realizar el «Paseo de la conciencia». Los comentarios de los niños no tenían desperdicio. (LHC, 1973 n)

La Gente del Pueblo Quiere

Por ahora, el Taller Comunitario celebrado en el Ayuntamiento de Yountville el pasado sábado ya forma parte de la historia.

Un centenar o más de nosotros pasamos desde las 10:00 hasta las 17:00 trabajando, argumentando, a veces discutiendo y finalmente escribiendo nuestras ideas e ilustrándolas en grandes hojas de papel para luego presentarlas oralmente delante de todo el grupo.

Me quedé impresionado con todo el programa, sobre todo porque se trataba de una muestra representativa de la comunidad de Yountville, personas mayores, veteranos de guerra, matrimonios jóvenes, solteros, profesionales e incluso asistieron no residentes.

También me deleité con los arquitectos empleados por la ciudad; Lawrence Halprin \& Asociados. El Sr. Halprin y su personal (once técnicos) estuvieron presentes durante toda la sesión. Estaban allí para escuchar los deseos de los ciudadanos, y escucharon. Escucharon con atención, cortesía, y estaban constantemente escribiendo notas sobre las presentaciones. La cooperación parecía ser la tónica del día. (LHC, 1973 ñ)

Como era de esperar también hubo críticas, alguna verdaderamente furibunda como la que escribió la periodista Pam Hunter en el periódico The register. En ella se acusa de pagar un altísimo precio por el taller participativo, que se cayó en las mismas trampas y escollos que en las discusiones del Ayuntamiento, que se trató de manera fútil el crecimiento de la ciudad, que los participantes habían hecho los paseos de la consciencia en coche, y que obviamente no se trató el problema de la vivienda. LH\&A le responden con una carta que va rebatiendo punto por punto cada una de sus diatribas, y que en resumen viene a recordar que sus objetivos para este taller eran bien conocidos. No se esperaba ni se pretendía que los ciudadanos de Yountville resolvieran sus problemas, que ésa es tarea de LH\&A, COMO Halprin había señalado en su introducción al taller. Se pretendía que establecieran cuáles eran sus deseos para Yountville, lo que esperaban de su evolución y las prioridades para su futuro; y esto se hizo con claridad y buen humor. 


\section{Fases IV-VII. La representación teatral como medio de transmisión de ideas en la planificación}

Previo a seguir trabajando en las siguientes fases, LH\&A produce un informe que entrega al Ayuntamiento donde describe los objetivos comunitarios de Yountville según lo observado-acordado en el Taller Participativo.

En este informe trasladan a términos arquitectónico-urbano técnicos las voluntades populares que aparecieron en el Taller Participativo. Los diferentes objetivos se agrupan en 3 grupos principales: Política de la Comunidad, Política del Suelo y Servicios Necesarios. Interesantísima la traducción del Sentir de la calle al Planeamiento, pero deberá ser objeto de explicación detallada en otro momento.

Al continuar el proceso después del taller, un dato a la vez entrañable y destacable es el comentario que la economista Linda Hausrath apunta en la carta donde se definen los términos del contrato que la consultoría económica $\mathrm{GG}^{+} \mathrm{A}$ ofrecerá a $\mathrm{LH} \& \mathrm{~A}$ en las siguientes fases, y que sorprende por su empatía al estar escrito al final de un texto de carácter económico pero a su vez nos indica la capacidad de convocatoria que tuvieron LH\&A y el Ayuntamiento $\left(\mathrm{GG}^{+} \mathrm{A}\right.$ tenía la sede en San Francisco y aunque ya habían contactado con anterioridad aún no estaba contratada) y el entusiasmo que conllevó dicha acción.

De nuevo he de decirle lo mucho que disfruté el taller en Yountville. Fue un gran éxito. (LHC, 1973 o)

El 17 de diciembre el secretario del ayuntamiento emplaza a LH\&A a reunirse en el Ayuntamiento para comentar las conclusiones del Taller Participativo y los nuevos plazos de entrega.

En los archivos de LH\&A consultados no se ha encontrado el registro de lo que se comentó en esa reunión, pero queda claro que los trabajos continuaron su marcha y como consecuencia de todo lo aportado en el taller más la reunión en el Ayuntamiento, el equipo de LH\&A se vio obligado a plantear distintas alternativas de planeamiento urbano. 
Es evidente que todas las ideas y voluntades no se podían llevar a la práctica con la misma intensidad, incluso había objetivos contradictorios, por lo que entonces surgió la necesidad de escoger.

Para informar a la población de las distintas opciones se aprovecha la fiesta anual que ofrece la Cámara de Comercio el 18 de enero (cena, baile, cata de vinos y desfile de moda), que se realiza en el Ayuntamiento, y se añade un nuevo punto en el programa del día; la representación teatral: 4 Alternativas Futuras para Yountville.

Se transcribe el guión del evento preparado en LH\&A:

INTRODUCCIÓN:

Larry (Lawrence Halprin) explicará lo que nos llevó a esta forma de presentación y compartirá con el público sus propios sentimientos personales acerca de las diversas alternativas. Pedirá a la audiencia específicamente no responder en este momento y mencionará una fecha u ocasión futura cuando se podrá hacer.

Les pedirá que sólo se concentren y disfruten de lo que escuchen y vean, explicando que tiene más sentido como entretenimiento, al menos en su foro interno, y que si hay un lado serio, éste va a aflorar en la creación de una imagen a través de su contenido.

(3 minutos)

\section{REPRESENTACIONES [fig. 4]}

Alternativa Futura \#1 - Preservar Yountville como un entorno natural.

Sin embargo... la preservación de una cosa puede resultar en el sacrificio de otra.

Alternativa Futura \#2 - Explotar el único atractivo de Yountville para el turismo.

Sin embargo... a veces uno se convierte en el esclavo de lo que explota.

Alternativa Futura \#3 - Maximizar el potencial de desarrollo en Yountville.

Sin embargo... maximizar la cantidad no significa necesariamente maximizar la calidad.

Alternativa Futura \#4 - Actualización y ampliación del entorno residencial en Yountville. Sin embargo... un pueblo pequeño no tiene por qué ser prospero o diverso. (LHC, 1973 p) 


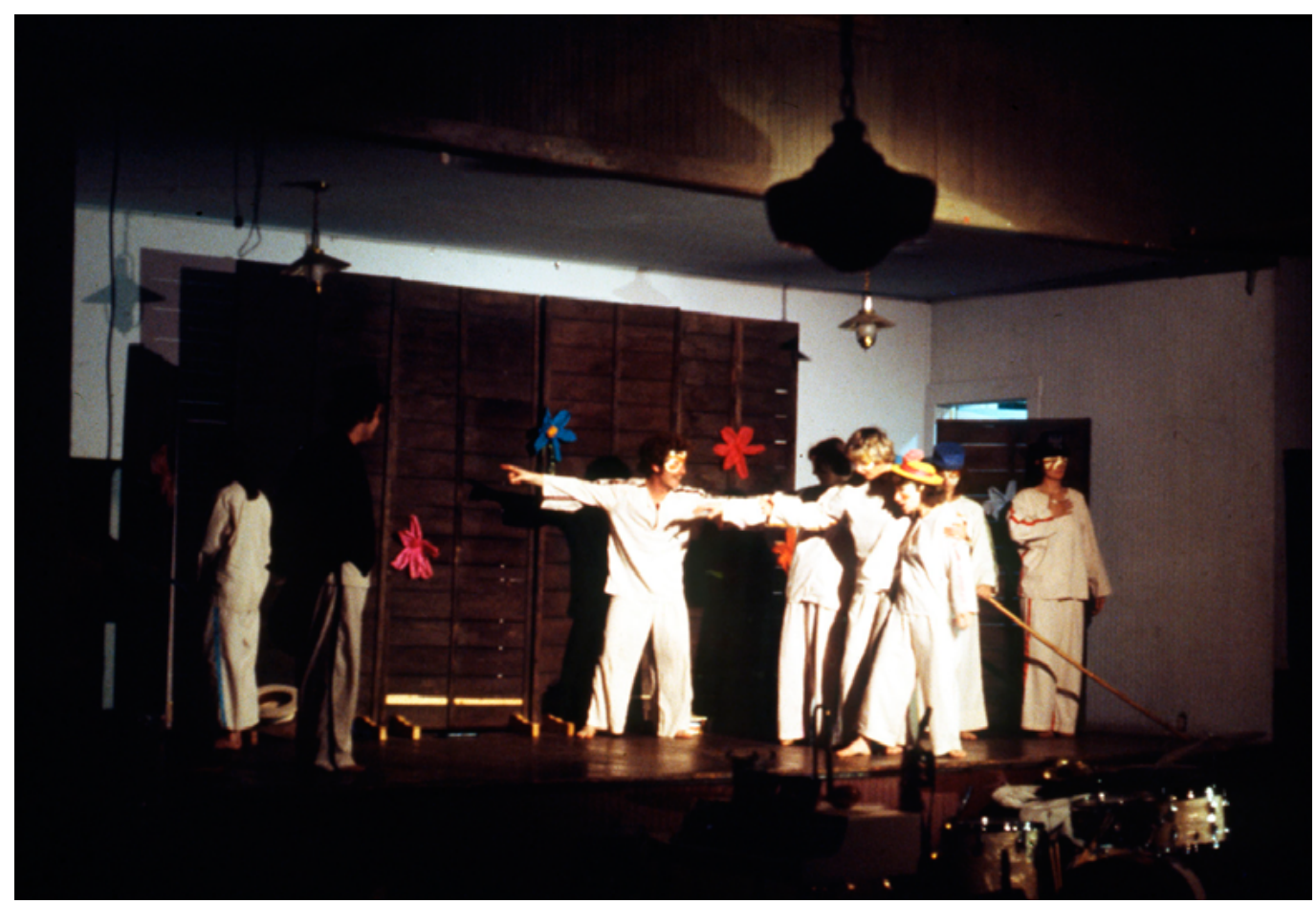

Figura 4. Imagen de la representación teatral: 4 Alternativas Futuras para Yountville, 1973. Fuente: Lawrence Halprin Collection, The Architectural Archives, University of Pennsylvania [Slide: 014.VI.HC-312]

En la crónica firmada por Glady Doolitle que apareció el 23 de enero en el Napa County Records se describe que había 201 comensales invitados. La obra de teatro fue producida por la compañía del Napa Valley Theatre en coordinación con LH\&A, y protagonizada por George C. Yount (el personaje que dio nombre a la ciudad, interpretado por el actor Dan Woodworth). Y al final de las representaciones se anunció que al cabo de un par de semanas se discutirían públicamente las 4 alternativas.

$\mathrm{Al}$ respecto de la obra de teatro documenta:

Cada una de las alternativas tiene ventajas, pero la responsabilidad de la elección de un plan para determinar el futuro de Yountville se encuentra en sus propios ciudadanos, comentó uno de los personajes de la obra.

El primer plan implicaba la «renovación interior» del parque de vivienda que haría que la ciudad se viera «bonita», aumentara las rentas y obligara a los alquilados a marchar. («Eso es muy triste», dijo uno de los personajes.) 
El segundo plan evolucionaba alrededor del pleno desarrollo de la pequeña aldea. El «rico» genera con la propiedad ingresos para la ciudad y Yountville se convierte en una gran ciudad.

La tercera alternativa demostraba cómo Yountville podía ser una «gran atracción turística» con la preservación de la ciudad («el pueblo más pintoresco e histórico que nadie haya pisado») y sin desarrollo comercial. Uno de los personajes dice de los turistas: « Gastan dinero y tendremos un montón de ingresos!».

El último plan parecía indicar una mezcla de desarrollo para atraer turistas y preservar la ciudad mediante la explotación de su «historicidad» y el medio natural. (LHC, 1973 q)

A principios de febrero se difunde el bando del Ayuntamiento con la convocatoria de la reunión para la discusión de las 4 alternativas y se ofrece la posibilidad de explicaciones suplementarias con los técnicos municipales durante la semana anterior al acto.

El 19 de febrero de 1974 LH\&A presentan públicamente mediante diapositivas y dibujos las 4 alternativas que se habían ofrecido en forma de obra teatral el 18 de enero. Se atienden dudas y preguntas y se habla de las ventajas y desventajas de cada una.

A principios de marzo y atendiendo a los resultados del primer taller (realizado el 13 de octubre de 1973), los comentarios realizados el día de la presentación (18 de enero) y la discusión de las 4 alternativas (19 de febrero de 1974) el comité de ciudadanos publica sus conclusiones al respecto de la alternativa que Yountville debería seguir.

La alternativa escogida es la de Preservación y Conservación, añadiendo algunos aspectos de otras alternativas como la voluntad de tener un pequeño centro comercial o una expansión limitada de los equipamientos turísticos. Se convoca a la ciudadanía a discutirlo el 19 de marzo para aprobarlo popularmente y trasladar la recomendación al gobierno municipal para que lo apruebe políticamente.

El panfleto utiliza exactamente la gráfica usada por la firma de LH\&A. Con la información consultada no se puede afirmar si directamente la Comisión ciudadana la copió, o es que se redactó desde LH\&A; las dos versiones son verosímiles. 
Después de la nueva discusión pública a la alternativa a seguir, el Ayuntamiento publica un resumen de cuatro páginas con las conclusiones a las que ha llegado y con los futuros objetivos urbanos definidos.

Dicha Declaración de Enfoque se comunica el 26 de marzo a LH\&A para que así pueda seguir avanzando en la realización del nuevo Plan Urbano de Yountville.

Por otro lado, la declaración también se hace pública para que los habitantes conozcan la propuesta consensuada en el Ayuntamiento y la comenten o cuestionen llamando al secretario o dejando nota escrita en el propio Ayuntamiento.

En esta declaración además de describir los objetivos urbanos globales, también se detallan los siguientes capítulos: Tráfico y Circulación, Espacio Abierto, Desarrollo Comercial, Desarrollo Residencial, Equipamientos, Conclusiones y cuál es el siguiente paso a seguir.

En el último punto se anota que en unas cuatro semanas (a finales de abril) LH\&A entregará un plano con el desarrollo planeado y cómo éste afectará a cada parcela de suelo municipal. Entonces se convocará un nuevo encuentro público para anotar problemas, dudas o cuestiones que surjan y cuando éstas se hayan resuelto los arquitectos dibujarán la versión final del Plan para que sea aprobado en el Ayuntamiento.

A inicios de junio de 1974 se anuncia la presentación del borrador del nuevo Máster Plan y para la semana siguiente se abre el periodo de consultas.

La comisión ciudadana convoca a la población de Yountville, para el 22 de Julio en el Ayuntamiento, como última instancia para aportar ideas o alegaciones previo a encargar a LH\&A la redacción definitiva del Plan a partir del día siguiente a esa reunión.

E1 23 de julio en sesión del Ayuntamiento son aprobadas por unanimidad las consideraciones y matices al borrador del Plan aportadas por el Comité de Ciudadanos y el 24 se ponen en conocimiento de LH\&A, para que las incorpore al Plan y redacte su versión definitiva.

El 7 de agosto LH\&A comunica al Ayuntamiento que se han atendido e incorporado al Plan la mayoría de las peticiones de la Comisión de Ciudada- 
nos pero se razonan unas cuestiones que afectan a un par de esas voluntades para que el Ayuntamiento reflexione si realmente se deben considerar. Para ello también les ofrece un par de sugerencias alternativas para resolver la cuestión que el Ayuntamiento tomará en consideración.

A partir de aquí sigue avanzando el proyecto de Plan Urbano según los tiempos previstos, incorporando alguna nueva petición o exigencia del gobierno supramunicipal.

El 15 de enero de 1975 se presenta en público el borrador final del Plan.

Y después de todos los trámites burocráticos con las diferentes administraciones locales, regionales y estatales y los tiempos de exposición pública prescriptivos se aprueba finalmente el Plan Urbano de Yountville, con fecha 1 de mayo de 1975.

\section{Discusión}

En todo este proceso de participación ciudadana, experimental en ese momento en EEUU, y muy poco aplicado en España aún a día de hoy medio siglo más tarde, se pueden destacar varios hitos significativos en el proceder de la definición de la planificación urbana.

\section{Se escucha al ciudadano.}

No solo para que vote una alternativa dada (que ya sería mucho en nuestras latitudes) sino para que él la proponga. El ciudadano no sólo es consultado sino que construye y conceptualiza la ciudad. Nos encontramos en uno de los niveles más altos en la Escalera de Participación Ciudadana de Arnstein (Arnstein, 1969).

\section{El paseo de la consciencia.}

Una actividad en la que se iguala a todos los ciudadanos para que tengan un denominador común de partida. Una experiencia particular y guiada de la ciudad, que los hace parar a reconocerla y reflexionar y pensar sobre ella. Al fin y al cabo ser conscientes del lugar donde viven. 


\section{El taller participativo como creación.}

Todo el mundo coopera de una manera u otra en unas propuestas de creación colectiva dirigidas a repensar la ciudad. Da igual la técnica que uno domine, cualquiera es capaz de expresar algo y aportarlo al grupo. Puede ser una poesía, un dibujo, una reflexión, un chiste, un collage, una redacción, una declamación..., todas las opciones son buenas y enriquecedoras.

\section{El taller participativo como convivencia.}

Al mezclar personas de distintas procedencias e intereses y empujarlos a crear juntos se establecen empatías que ayudan a comprender al otro. Se pierden los roles del poder, nadie manda o impone por encima de otro. El grupo equilibra, y no es cuestión de que alguien gane en contraprestación de que otro pierda, sino que la escucha compartida ayuda a crear consensos en los que el grupo siempre gana.

\section{El teatro como herramienta de reflexión.}

La representación teatral que caricaturizaba 4 alternativas de ciudad planteada se realiza semanas después de que todo el mundo ya hubiera hecho unas mínimas reflexiones sobre la ciudad.

La actuación tiene lugar un día de fiesta popular, sin permitir la discusión posterior con los arquitectos o el Ayuntamiento. Pero allí se libera la pregunta discordante que promete resolverse en un par de semanas.

En el taller fueron todo ideas positivas, un juego cívico placentero y festivo, ahora sin dejar el civismo toca decidir y descartar con responsabilidad. El teatro es usado en este proceso como un revulsivo para la reflexión.

Cuando más tarde se dan las explicaciones pertinentes de las 4 propuestas, éstas están mucho más elaboradas de lo que se intuía, acompañadas de datos históricos, geográficos, económicos, etc., pero son fáciles de comprender y discutir con razones, después del bagaje acumulado.

\section{6. ¿Quién proyecta?}

Es muy destacable la decisión que realiza el Ayuntamiento al decantarse por la que consideran la mejor propuesta y no la más económica. Si compara- 
mos con los concursos actuales que licitan proyectos semejantes, en muchos casos la valoración económica pesa un $70 \%$ respecto al $30 \%$ de la técnica. Y podríamos considerar que ésta no es una ponderación correcta, ya que prima la miope valoración de la propuesta económica del profesional por encima de su capacidad, aunque luego la institución sea incapaz de controlar el desvío implacable de fondos inherentes en el desarrollo de muchas de esas supuestas bajas económicas. Más injusta y desproporcionada es si cabe este tipo de valoración cuando el coste del profesional es ínfimo al lado del coste de la puesta en práctica del servicio por el cual se ha contratado, que quizás luego nadie fiscaliza. Por lo que al final la sociedad acaba pagando un precio altísimo por un trabajo de menor calidad.

\section{Aplicación hoy.}

Conociendo el origen de los procesos participativos y la metodología de los ejemplos desarrollados con éxito, en cualquier momento se pueden aplicar adaptados a otro tiempo y condición.

\section{Conclusiones}

La participación ciudadana en la construcción de la ciudad es una acción intrínsecamente positiva para la ciudadanía, ya que ayuda a aproximar el resultado de la construcción urbana a los intereses generales, y no a los de una élite minoritaria. Es decir, se busca la creación de un bien común.

Por otro lado y de forma colateral, el hecho de la participación de un grupo representativo y transversal de la sociedad provoca que el sentimiento creativo en la consecución de la definición de la forma urbana se convierta en un sentimiento de identidad y pertenencia con lo nuevo proyectado; facilitando la comprensión y aceptación de las decisiones que el consistorio pueda aprobar si siguen la misma línea del interés general.

\section{Referencias}

Arnstein, S. R. (1969): «A ladder of citizen participation». En: Journal of the American Institute of Planners, 35 (4): 216-224. Disponible en $<$ http://lithgow-schmidt.dk/sherry-arnstein/ladder-of-citizen-participation_en.pdf $>$. 
Blancafort, J. y P. Reus (2015): «Pioneros de la participación colectiva en los procesos de planificación urbana. Legado Halprin». En: $A C E$, Architecture, City and Environment, 38: 57. Disponible en $<\mathrm{http} / /$ upcommons.upc.edu/bitstream/handle/2099/16652/3681-1231-1PB.pdf? sequence $=1 \&$ isAllowed $=\mathrm{y}>$.

Halprin, L. \& Associates (1972 a): Take Part: A report on new ways in which people can participate in planning their own environments. Lawrence Halprin \& Associates.

- (1972 b): Ibíd.

Lawrence Halprin Collection (lhc), The Architectural Archives, University of Pennsylvania (1972): Folder [014.I.A.3559] 1972-1103 Memorandum GGa. Gruen Gruen + Associates.

— (1973 a): Folder [014.I.A.3559] 1973-09-05 Data Planning Department. Development and Planning Department, County of Napa.

— (1973 b): Folder [014.I.A.5623] 1972-11-09 Being considered. Letter from Barry L. Wasserman (Project Manager LH\&A) to Robert Keenan (City of Yountville).

— (1973 c): Folder [014.I.A.3559] 1973-06-15 Information received from Yountville City Council.

— (1973 d): Folder [014.I.A.5623] 1973-07-06 Plan Contract.

- (1973 e): Ibid.

— (1973 f): Folder [014.I.A.3559] 1973-09-05 Letter to Barry from Steve Bardessono (City Council).

— (1973 g): Folder [014.I.A.3559] 1973-09-25 Letter from Mayor (Don Schmitt) to citizens.

— (1973 h): Folder [014.I.A.3559] 1973-10-09 Letter from Mayor.

— (1973 i): Folder [014.I.B.1250] 1973-09-10 Field Trip for Yountville Workshop. Awareness Walk-ride Score.

— (1973 j): Folder [014.I.B.1250] 1973-10-12? Final Agenda Score.

— (1973 k): Folder [014.I.B.1250] 1973-10-13 Script.

—(1973 1): Ibid.

— (1973 m): Folder [014.I.A.3559] 1973-10-17 Clipping. Welcome to Yountville. Napa County Records.

— (1973 n): Folder [014.I.A.3559] 1973-10-17 Clipping. Awareness day. Mayor's view by Don Schmitt. Napa County Records.

—(1973 ñ): Folder [014.I.A.3559] 1973-10-17 Clipping. The Town People Want, by Glady Doolitle. Napa County Records.

— (1973 o): Folder [014.I.A.3559] 1973-11-01 Agreement with GGa. 
— (1973 p): Folder [014.I.B.1250] 1974-01-18 Annual Chamber Dinner.

- (1973 q): Folder [014.I.B.1250] 1974-01-23 Clipping-Chamber Dinner. The Plan was a Play for a Night, by Glady Doolitle. Napa County Records. 